\title{
Progressive systemic sclerosis in the elderly
}

\author{
J. A. DALZIEL* \\ M.A., M.R.C.P.
}

\author{
G. K. WILCOCK \\ D.M., M.R.C.P.
}

\author{
Department of Geriatric Medicine, The Cowley Road Hospital, Oxford
}

\begin{abstract}
Summary
There is evidence to suggest that the incidence of progressive systemic sclerosis in the elderly is more common than in younger age groups. As in younger patients, late onset cases may have minimal skin changes and this can cause difficulty in diagnosis. The case histories of 2 patients are presented to illustrate these points.
\end{abstract}

\section{Introduction}

Although there is no universally agreed classification of scleroderma, the following is most generally accepted (Rowell, 1977):

(i) morphoea (localized or generalized); (ii) progressive systemic sclerosis; (iii) pseudo scleroderma; (iv) occupational scleroderma.

Progressive systemic sclerosis usually begins in middle age, but there is a wide range in the age of onset. Rodnan (1963) found that the incidence by age at diagnosis increases steadily, reaching a peak in the over 65 years age group. The findings of Medsger and Masi (1971) support this view.

Clinically detectable changes in internal organs usually appear several years after the skin manifestations; however, visceral involvement may occur first and the diagnosis may be overlooked until skin changes appear. In some cases there has been severe systemic involvement with only minor cutaneous changes (Rodnan and Fennell, 1962).

The following case histories illustrate the need to consider this condition in elderly patients despite the absence of typical skin changes, especially if there is a history of Raynaud's phenomenon.

\section{Example cases}

No. 1

An 87-year-old woman, when admitted for management of cardiac failure, was found to have a long history of Raynaud's phenomenon and pains in the joints of both hands. She had no dysphagia. She presented arthritic changes in both hands involving many of the small joints, together with loss of finger pulp in the left index and middle fingers. Similar

\footnotetext{
* Present address: Department of Geriatric Medicine, Leicester General Hospital, Leicester.
}

joint changes were present in the feet. There was also generalized small muscle atrophy and the skin of the fingers was somewhat tethered to the underlying tissue but there was no obvious sclerodactyly. Facial telangiectasia was also noted.

There were no other skin lesions either on admission or subsequent follow-up over 5 months. The signs of cardiac failure improved with an increase in diuretic therapy and addition of digoxin although she remained somewhat dyspnoeic.

Investigations. Blood count, ESR, biochemical screen, protein electrophoresis and MSU, were all normal. Antinuclear factor, LE cells, and Rose Waaler test were negative, but the latex screening test was positive, and smooth muscle antibodies were detected at a titre of $1 / 20$.

Chest X-ray showed cardiomegaly and pulmonary oedema and the ECG ischaemic changes. X-ray of her hands showed extensive soft tissue calcification, minimal erosive changes, and arthritic changes in the small joints. A barium swallow revealed a short, dilated aperistaltic oesophagus.

\section{No. 2}

An 85-year-old woman, when admitted for treatment for leg ulceration, was noted to have difficulty in swallowing and the symptoms of Raynaud's phenomenon.

Examination revealed mat-like telangiectases on her face and hands including palms, tethering of the skin of her fingers and a pinched facial appearance. She had widespread osteoarthritis and also an arthropathy involving the distal interphalangeal joints.

Investigations. Routine investigations including $\mathrm{Hb}, \mathrm{ESR}$, urea and biochemical screen were normal apart from a raised MCV due to folate deficiency. Tests for malabsorption were negative. Tests for ANF and rheumatoid factor were also negative.

Chest X-ray was within normal limits. X-ray of hands revealed arthritic changes of rheumatoid type including distal interphalangeal joints. Barium swallow revealed a poorly co-ordinated pharyngeal phase of swallowing with decreased peristalsis, and delay at the cardio-oesophageal sphincter. 


\section{D̄iscussion}

The patients described here did not present with the classical skin changes of scleroderma but they did have some tethering of the skin of the fingers and mat-like telangiectases which are features of scleroderma. In the authors' experience skin tethering does not appear to be common in patients with no other evidence of scleroderma, although telangiectases are frequently seen in the elderly exposed skin. Both patients had Raynaud's phenomenon and this is almost universal in systemic sclerosis (Rowell, 1977). Le Roy (1976) considers that a high proportion of patients with Raynaud's phenomenon when followed-up carefully for several years eventually develop systemic sclerosis. In view of this, the authors are currently studying a third patient with long-standing Raynaud's phenomenon who has a distal arthropathy of her hands together with skin tethering and telangiectases, since she may develop further signs of systemic sclerosis.

Both patients had arthropathy of the distal joints of both hands. Joint limitation in advanced cases of systemic sclerosis with skin involvement is related to the hardening of the skin and underlying tissues, but a true polyarthritis may occur early in the disease involving mainly small joints (Rodnan, 1962). Although it is possible to have coincident rheumatoid arthritis and scleroderma, such cases are not numerous (Jablonska, 1975) and there is no reason to suppose this was the case in the above patients. In the majority of Jablonska's cases joint changes of rheumatoid arthritis preceded the appearance of the skin and visceral signs of systemic sclerosis. The presence of rheumatoid factor in case no. 1 does not imply rheumatoid arthritis since rheumatoid factor is found in about $33 \%$ of cases of systemic sclerosis. Pseudo-sclerodermatous skin changes may occur in rheumatoid arthritis but Raynaud's phenomenon is considered to be rare (Jablonska, 1975).

Both patients had oesophageal changes and it is interesting that the first patient, with the most extensive involvement, had no symptoms from this.

The oesophagus is involved in systemic sclerosis so frequently that Garrett et al. (1971) suggested that the physician should be reluctant to make the diagnosis without evidence of oesophageal involvement. Early cases show weak pressures and inco-ordinated contractions in the smooth muscle and this progresses to shortening, stricture and dilatation. There is gradual weakening of the sphincter together with reflux and more than $50 \%$ of sufferers have hiatus hernia.

Patients with involvement of the small bowel may present with malabsorption (McBrian and Mummery, 1962) and this was suspected in the second patient because of her folate deficiency, but not confirmed on further investigation. Although involvement of the heart is frequent in systemic sclerosis, in case no. 1 the cardiac failure was more likely to be due to ischaemic heart disease since there was a good response to treatment (Askanas, Slucka and Ceremuzynske, 1975). Her persistent dyspnoea, however, may have been due to pulmonary involvement; unfortunately she refused pulmonary function tests, but these usually show a restrictive pattern with reduced transfer factor. Muscle involvement is not widely recognized but Medsger et al. (1968) found that the majority had evidence of a myopathy in particular involving the shoulder girdle.

Although most cases are diagnosed in middle age, epidemiological studies (Rodnan, 1962; Medsger and Masi, 1971) suggest that the incidence of systemic sclerosis reaches a peak in the over 65 years age group. However, whilst not a common condition, it is rarely considered in elderly patients, leading to under-diagnosis. There may be several reasons for this. The skin changes may be overlooked either because they are minimal, possibly restricted to mild tethering of skin in the hands, or because they are misinterpreted as features of ageing, for example radial furrowing around the mouth. In the absence of skin changes the diagnosis is less frequently considered especially in the elderly in whom multiple symptoms such as dysphagia, Raynaud's phenomenon and arthralgia can more frequently be attributable to multiple pathologies rather than a multi-system disorder.

\section{References}

Askanas, Z., Slucka, C. \& Ceremuzynske, L. (1975) Visceral scleroderma. In: Scleroderma and Pseudoscleroderma (Ed. by Jablonska, S.), p. 311. Dowden, Hutchinson \& Rose, Inc., Stroudsburg, Pennsylvania.

Garrett, J.M., WinkelmanN, R.K., Schlegel, J.F. \& Code, C.F. (1971) Esophageal deterioration in scleroderma. Proceedings. Mayo Clinic, 46, 92.

JABLONSKA, S. (1975) Scleroderma-like lesions in rheumatoid arthritis. In: Scleroderma and Pseudoscleroderma (Ed. by Jablonska, S.), p. 461. Dowden, Hutchinson \& Rose Inc., Stroudsburg, Pennsylvania.

Le Roy, E.C. (1976) Scleroderma. In: Topics in Rheumatology. (Ed. by Hughes, G.R.V.). Heinemann, London.

McBrian, D.J. \& Mummery, H.E.L. (1962) Steatorrhoea in progressive systemic sclerosis. British Medical Journal, 2, 1653.

Medsger, T.A., Rodnan, G.P., Moossy, J. \& Vester, J.W. (1968) Skeletal muscle involvement in progressive systemic sclerosis. Archives of Rheumatology, 11, 554.

MedsGer, T.A. \& MASI, A.T. (1971) Epidemiology of systemic sclerosis (scleroderma). Annals of Internal Medicine, 74, 714.

RodNAN, G.P. (1962) The nature of joint involvement in progressive systemic sclerosis. Annals of Internal Medicine, 56, 422.

RoDnan, G.P. (1963) A review of recent observations and current theories on the etiology and pathogenesis of progressive systemic sclerosis (diffuse scleroderma). Journal of Chronic Diseases, 16, 929.

Rodnan, G.P. \& Fennell, R.H. (1962) Progressive systemic sclerosis sine scleroderma. Journal of the American Medical Association, 180, 665.

Rowell, N. (1977) Scleroderma. Practitioner, 219, 820. 